Website: ijetms.in Issue: 7 Volume No.4 November - 2020 DOI: 10.46647/ijetms.2020.v04i07.001

\title{
Application of Non-Linear Programming to Portfolio Management on some Insurance Companies (AIICO, LINKAGE, NIGER, Mutual benefit and LASACO) using Return on Asset
}

\author{
Olawale Kolapo Steve Emiola ${ }^{1}$, Musibau Abayomi Omoloye ${ }^{2}$, Christiana Uchechukwu Arinze ${ }^{3}$ \\ ${ }^{1,2,3}$ Department of Statistics, Federal Polytechnic Offa, Kwara State, Nigeria. \\ ${ }^{1}$ Stevemiola@ fedpoffaonline.edu.ng \\ 2omoloye2011@gmail.com
}

\begin{abstract}
This study investigate non-linear programming problem that is, quadratic programming and its application to portfolio management. The data of return on asset of five different insurance companies namely: AIICO, LINKAGE, NIGER, MUTUAL BENEFIT, and LASACO insurance company was collected and a model was fixed. These data were analyzed using quadratic programming in conjunction with LINGO software. The result of the analyzed data revealed that the allocation of fund for each insurance companies should be done with the same percent for LINKAGE, NIGER, MUTUAL BENEFIT and other percent to AIICO insurance company respectively with increment of $24 \%$ on return.
\end{abstract}

Keywords - Return on assets, insurance companies, portfolio management and model.

\section{INTRODUCTION}

Portfolio models are concerned with investment where there are typically two criteria: expected return and risk. The investor wants the former to be high and the latter to be low. There is a variety of measures of risk. The most popular measure of risk has been variance in return introduced by [12]. Quadratic programming is computationally appealing because the algorithms for linear programs can be applied to quadratic programming with only modest modifications. Loosely speaking, the reason only modest modification is required is the first derivative of a quadratic function is a linear function. Because LINGO has a general nonlinear solver, the limitation to quadratic functions is helpful, but not crucial.

Quadratic programming is a unique case of mathematical optimization problem. It is the problem of maximizing or minimizing a quadratic function where the variables are subject to linear constraints. Quadratic programming is used in portfolio optimization for the formulation of the mean-variance optimization of investments judgments under uncertainty.
Portfolio management, for an investor that has different assets to trade in, is choosing optimal investments, that is, how many shares of an asset should he/she buy and hold for him/her to maximize some criteria depending on his/her total wealth and/or consumption.

Return on assets (ROA) is a financial ratio that shows the percentage of profit a company earns in relation to its overall resources. It is commonly defined as net income divided by total assets. Net income is derived from the income statement of the company and it is the profit after taxes. The assets are read from the balance sheet and include cash and cash equivalent items such as receivables, inventories, land, capital equipment as depreciated, and the value of intellectual property such as patents.

Portfolio is a collection or an aggregation of investments tools such as stocks, shares, mutual funds, bonds, cash etc [4]. It also indicate that the decision of future yet unknown is premise on the information gotten from the past.

[5] Used return on invested capital to investigate how much Dangote can invest on three of his subsidiaries Viz. Dangote Cement, Dangote Sugar refinery and Dangote Flour given an amount available to him.

[1] Used the quadratic approach to select the optimum portfolio of the Malaysian stock exchange and her framework deals with ten biggest firms posted on the stock exchange during 2014. The result shows that the optimum portfolio includes $22 \%$ of Axiata Group shares, $11 \%$ of Genting shares, $30 \%$ of Petronas Chemicals shares, $1 \%$ of Sime Darbi shares and $36 \%$ of Tenaga Nasional shares.

[6] In his thesis used dividend payout ratio as a determinant to investigate how to make selection of Bank shares in three different Banks, which are Zenith Bank, Guaranty Trust Bank plc, and First Bank plc.

However, this study is working on five (5) different insurance companies which are AIICO Insurance Company, Linkage Insurance Company, LASACO Insurance Company, Niger Insurance Company and Mutual Benefit Insurance Company to investigate the percentage to invest on each company's return on asset. 


\section{International Journal of Engineering Technology and Management Sciences}

Website: ijetms.in Issue: 7 Volume No.4 November - 2020 DOI: 10.46647/ijetms.2020.v04i07.001

\section{DATA PRESENTATION}

For the purpose of this work, abstraction from established published sources was used to collect data. The data used was already in existence but were extracted for the purpose of this work and explained briefly below.

The table1: below shows the Percentage Return on Asset invested on Linkage insurance company, Mutual Benefit insurance company, Niger insurance company, AIICO insurance company and LASACO insurance company.

\begin{tabular}{|c|c|c|c|c|c|c|c|c|c|c|}
\hline $\begin{array}{l}\text { Insura } \\
\text { nce } \\
\text { comp } \\
\text { any }\end{array}$ & $\begin{array}{l}20 \\
08\end{array}$ & $\begin{array}{l}20 \\
09\end{array}$ & $\begin{array}{l}20 \\
10\end{array}$ & $\begin{array}{l}20 \\
11\end{array}$ & $\begin{array}{l}20 \\
12\end{array}$ & $\begin{array}{l}20 \\
13\end{array}$ & $\begin{array}{l}20 \\
14\end{array}$ & $\begin{array}{l}20 \\
15\end{array}$ & $\begin{array}{l}20 \\
16\end{array}$ & $\begin{array}{l}20 \\
17\end{array}$ \\
\hline $\begin{array}{l}\text { LINK } \\
\text { AGE }\end{array}$ & $\begin{array}{l}2 . \\
8\end{array}$ & $\begin{array}{l}3 . \\
1\end{array}$ & $\begin{array}{l}3 . \\
9\end{array}$ & $\begin{array}{l}4 . \\
2\end{array}$ & $\begin{array}{l}4 . \\
4\end{array}$ & $\begin{array}{l}3 . \\
7\end{array}$ & $\begin{array}{l}3 . \\
9\end{array}$ & $\begin{array}{l}3 . \\
9\end{array}$ & $\begin{array}{l}3 . \\
9\end{array}$ & $\begin{array}{l}3 . \\
3\end{array}$ \\
\hline $\begin{array}{l}\text { MUT } \\
\text { UAL } \\
\text { BENE } \\
\text { FIT }\end{array}$ & $\begin{array}{l}2 . \\
5\end{array}$ & $\begin{array}{l}2 . \\
0\end{array}$ & $\begin{array}{l}3 . \\
1\end{array}$ & $\begin{array}{l}3 . \\
5 .\end{array}$ & $\begin{array}{l}3 . \\
0\end{array}$ & $\begin{array}{l}3 . \\
0\end{array}$ & $\begin{array}{l}3 . \\
6\end{array}$ & $\begin{array}{l}3 . \\
3\end{array}$ & $\begin{array}{l}2 . \\
8\end{array}$ & $\begin{array}{l}2 . \\
7\end{array}$ \\
\hline $\begin{array}{l}\text { NIGE } \\
\mathrm{R}\end{array}$ & $\begin{array}{l}0 . \\
2\end{array}$ & $\begin{array}{l}1 . \\
2\end{array}$ & $\begin{array}{l}1 . \\
8\end{array}$ & $\begin{array}{l}. \\
1\end{array}$ & $\begin{array}{l}1 . \\
4\end{array}$ & $\begin{array}{l}2 . \\
4\end{array}$ & $\begin{array}{l}1 . \\
5\end{array}$ & $\begin{array}{l}2 . \\
1\end{array}$ & $\begin{array}{l}2 . \\
1\end{array}$ & $\begin{array}{l}2 . \\
3\end{array}$ \\
\hline $\begin{array}{l}\text { AIIC } \\
\text { O }\end{array}$ & $\begin{array}{l}2 . \\
2\end{array}$ & $\begin{array}{l}3 . \\
7\end{array}$ & $\begin{array}{l}2 . \\
6\end{array}$ & $\begin{array}{l}2 . \\
2\end{array}$ & $\begin{array}{l}0 . \\
9\end{array}$ & $\begin{array}{l}2 . \\
6\end{array}$ & $\begin{array}{l}0 . \\
7\end{array}$ & $\begin{array}{l}. \\
3\end{array}$ & $\begin{array}{l}2 . \\
1\end{array}$ & $\begin{array}{l}. \\
1\end{array}$ \\
\hline $\begin{array}{l}\text { LASA } \\
\text { CO }\end{array}$ & $\begin{array}{l}2 . \\
6\end{array}$ & $\begin{array}{l}3 . \\
1\end{array}$ & $\begin{array}{l}3 . \\
6\end{array}$ & $\begin{array}{l}3 . \\
1\end{array}$ & $\begin{array}{l}2 . \\
4\end{array}$ & $\begin{array}{l}2 . \\
0\end{array}$ & $\begin{array}{l}1 . \\
8\end{array}$ & $\begin{array}{l}2 . \\
3\end{array}$ & $\begin{array}{l}3 . \\
1\end{array}$ & $\begin{array}{l}1 . \\
6\end{array}$ \\
\hline
\end{tabular}

Source: Annual financial record on Return on asset of five (5) selected insurance companies.

\section{DATA ANALYSIS}

An investor has fixed sum of money say $\mathrm{K}$, to invest in five (5) insurance companies namely; Linkage, Mutual Benefit, Niger, AIICO and LASACO.

The Portfolio problem is to determine how much money the investor should allocate to each insurance company so that total expected return is greater than or equal to some lowest acceptable amount say $\mathrm{T}$, and so that the total variance of future payment is minimized. to be

Let $\mathrm{X}_{1}, \mathrm{X}_{2}, \mathrm{X}_{3}, \mathrm{X}_{4}, \mathrm{X}_{5}$ designate the amount of money

allocated to Linkage insurance company, Mutual Benefit insurance company, Niger insurance company, AIICO insurance company, and LASACO insurance company respectively and let $\mathrm{X}_{\text {is }}$ denote the return per naira invested from invested from the investment $\mathbf{i}(\mathbf{i}=\mathbf{1}, \mathbf{2}, \mathbf{3}, \mathbf{4}$, 5) during the $S$ period of time in the past $(S=\mathbf{1}, \mathbf{2}, \mathbf{3}, \ldots$ 10). If the past history on return on asset is indicative of future performance, the expected future return per Naira from investment $1,2,3,4,5$ is

$$
\mathrm{E}_{\mathrm{i}}=\frac{\sum_{x=x 1}^{10} \text { bis }}{10}
$$

And the expected return from five investments combines is

$\mathrm{E}=\mathrm{E}_{1} \mathrm{X}_{1}+\mathrm{E}_{2} \mathrm{X}_{2}+\mathrm{E}_{3} \mathrm{X}_{3}+\mathrm{E}_{4} \mathrm{X}_{4}+\mathrm{E}_{5} \mathrm{X}_{5}$

The portfolio problem modeled as quadratic programming is

$$
\text { Min } \mathrm{R}=\mathrm{A}^{\mathrm{T}} \mathrm{CA}
$$

Subject to: $X_{1}+X_{2}+X_{3}+X_{4}+X_{5}=N$

$E X_{1}+E X_{2}+E X_{3}+E X_{4}+E X_{5} \geq K$

$X_{1} \geq 0, X_{2} \geq 0, X_{3} \geq 0, X_{4} \geq 0, X_{5} \geq 0$, where $C$ is the covariance matrix which is positive semi - definite i.e.

Matrix $\mathrm{C}=\left(\begin{array}{lllll}\sigma_{11}^{2} & \sigma_{12}^{2} & \sigma_{13}^{2} & \sigma_{14}^{2} & \sigma_{15}^{2} \\ \sigma_{21}^{2} & \sigma_{22}^{2} & \sigma_{23}^{2} & \sigma_{24}^{2} & \sigma_{25}^{2} \\ \sigma_{31}^{2} & \sigma_{32}^{2} & \sigma_{33}^{2} & \sigma_{34}^{2} & \sigma_{35}^{2} \\ \sigma_{41}^{2} & \sigma_{42}^{2} & \sigma_{43}^{2} & \sigma_{44}^{2} & \sigma_{45}^{2} \\ \sigma_{51}^{2} & \sigma_{52}^{2} & \sigma_{53}^{2} & \sigma_{54}^{2} & \sigma_{55}^{2}\end{array}\right), \mathrm{E}$ is the

mathematical expectation, A is column Matrix $=\left(\begin{array}{l}X_{1} \\ X_{2} \\ X_{3} \\ X_{4} \\ X_{5}\end{array}\right)$

Subject to: $\mathrm{X}_{1}+\mathrm{X}_{2}+\mathrm{X}_{3}+\mathrm{X}_{4}+\mathrm{X}_{5}=\mathrm{N}$

$$
\mathrm{EX}_{1}+\mathrm{EX}_{2}+\mathrm{EX}_{3}+\mathrm{EX}_{4}+\mathrm{EX}_{5} \geq \mathrm{K}
$$

$X_{1} \geq 0, X_{2} \geq 0, X_{3} \geq 0, X_{4} \geq 0, X_{5} \geq 0$.

Hence obtain

$$
\begin{aligned}
& \text { Min } \mathrm{R}=\mathrm{x}_{1}^{2} \sigma_{11}^{2}+\mathrm{x}_{2}^{2} \sigma_{22}^{2}+\mathrm{x}_{3}^{2} \sigma_{33}^{2}+\mathrm{x}_{4}^{2} \sigma_{44}^{2}+ \\
& \mathrm{x}_{5}^{2} \sigma_{55}{ }^{2}+\mathrm{X}_{1} \mathrm{X}_{2}\left(\sigma_{21}{ }^{2}+\sigma_{12}{ }^{2}\right)+\mathrm{X}_{1} \mathrm{X}_{3}\left(\sigma_{31}{ }^{2}+\sigma_{13}{ }^{2}\right) \\
& +\mathrm{X}_{1} \mathrm{X}_{4}\left(\sigma_{41}{ }^{2}+\sigma_{14}{ }^{2}\right)+\mathrm{X}_{1} \mathrm{X}_{5}\left(\sigma_{51}{ }^{2}+\sigma_{15^{2}}\right)+ \\
& \mathrm{X}_{2} \mathrm{X}_{3}\left(\sigma_{32}{ }^{2}+\sigma_{23}{ }^{2}\right)+\mathrm{X}_{2} \mathrm{X}_{4}\left(\sigma_{42}{ }^{2}+\sigma_{24}{ }^{2}\right)+\mathrm{X}_{2} \mathrm{X}_{5} \\
& \left(\sigma_{52}{ }^{2}+\sigma_{25}{ }^{2}\right)+\mathrm{X}_{3} \mathrm{X}_{4}\left(\sigma_{43}{ }^{2}+\sigma_{34}{ }^{2}\right)+\mathrm{X}_{3} \mathrm{X}_{5}\left(\sigma_{53}{ }^{2}+\right. \\
& \left.\sigma_{35^{2}}\right)+\mathrm{X}_{4} \mathrm{X}_{5}\left(\sigma_{54}^{2}+\sigma_{45^{2}}\right)+\left(\mathrm{X}_{1}+\mathrm{X}_{2}+\mathrm{X}_{3}+\mathrm{X}_{4}\right. \\
& \left.+\mathrm{X}_{5}-1\right) \mathrm{UNITY}+\left[1.20-\left(\mathrm{E}_{\mathrm{x} 1} \mathrm{X}_{1}+\mathrm{E}_{\mathrm{x} 2} \mathrm{X}_{2}+\right.\right. \\
& \mathrm{E}_{\mathrm{x} 3} \mathrm{X}_{3}+\mathrm{Ex}_{4} \mathrm{X}_{4}+\mathrm{E}_{\mathrm{x} 4} \mathrm{X}_{4}+\mathrm{E}_{\mathrm{x} 5} \mathrm{X}_{5} \mathrm{RETURN}+( \\
& \left.\mathrm{X}_{1}-0.25\right) \mathrm{X}_{1} \mathrm{FRAC}+\left(\mathrm{X}_{2}-0.25\right) \mathrm{X}_{2} \mathrm{FRAC}+( \\
& \left.\mathrm{X}_{3}-0.25\right) \mathrm{X}_{3} \mathrm{FRAC}+\left(\mathrm{X}_{5}-0.25\right) \mathrm{X}_{5} \text { FRAC }
\end{aligned}
$$

CALCULATED COVARIANCE MATRIX FROM MINITAB 


\section{International Journal of Engineering Technology and Management Sciences}

Website: ijetms.in Issue: 7 Volume No.4 November - 2020 DOI: 10.46647/ijetms.2020.v04i07.001

Matrix

$$
\mathrm{C}=\left(\begin{array}{ccccc}
0.25 & 0.18 & 0.12 & -0.20 & 0.03 \\
0.18 & 0.23 & 0.07 & -0.27 & -0.06 \\
0.12 & 0.07 & 0.45 & -0.11 & -0.13 \\
-0.20 & -0.27 & -0.11 & 0.87 & 0.40 \\
-0.03 & -0.06 & -0.13 & 0.40 & 0.43
\end{array}\right)
$$

Expected returns of return of asset for each insurance companies are $3.71 \%, 2.95 \%, 1.61 \%, 1.94,2.56 \%$ respectively. The budget constraint investment portfolio optimization problem has five candidate assets $\left(\mathrm{X}_{1}, \mathrm{X}_{2}, \mathrm{X}_{3}\right.$, $\mathrm{X}_{4}, \mathrm{X}_{5}$ ) for our portfolio.

\section{A. MODEL}

Determine what fraction should be devoted (or of the return on asset that the investor should have) to each insurance company, so an expected return of at least $25 \%$ (equivalently, a growth factor 1.25) is obtained while minimizing the variance in return and not exceeding a budget constraint.

It also impose a restriction that any given assets can constitute at most $25 \%$ of the portfolio. The variance if the entire portfolio is;

$\mathrm{R}=0.25 \mathrm{X}_{1}^{2}+0.23 \mathrm{X}_{2}^{2}+0.45 \mathrm{X}_{3}^{2}+0.87 \mathrm{X}_{4}^{2}+0.43 \mathrm{X}_{5}^{2}+$ $0.18 X_{1} X_{2}+0.12 X_{1} X_{3}-0.20 X_{1} X_{4}+0.03 X_{1} X_{5}+0.07 X_{2} X_{3}$ $-0.27 \mathrm{X}_{2} \mathrm{X}_{4}-0.06 \mathrm{X}_{2} \mathrm{X}_{5}-0.11 \mathrm{X}_{3} \mathrm{X}_{4}-0.13 \mathrm{X}_{3} \mathrm{X}_{5}+$ $0.40 \mathrm{X}_{4} \mathrm{X}_{5}$

Since variance is a measure of risk, we need to minimize, Hence

MIN R $=0.25 \mathrm{X}_{1}^{2}+0.23 \mathrm{X}_{2}^{2}+0.45 \mathrm{X}_{3}^{2}+0.87 \mathrm{X}_{4}^{2}+0.43 \mathrm{X}_{5}^{2}$ $+0.18 \mathrm{X}_{1} \mathrm{X}_{2}+0.12 \mathrm{X}_{1} \mathrm{X}_{3}-0.20 \mathrm{X}_{1} \mathrm{X}_{4}+0.03 \mathrm{X}_{1} \mathrm{X}_{5}+$ $0.07 \mathrm{X}_{2} \mathrm{X}_{3}-0.27 \mathrm{X}_{2} \mathrm{X}_{4}-0.06 \mathrm{X}_{2} \mathrm{X}_{5}-0.11 \mathrm{X}_{3} \mathrm{X}_{4}-0.13 \mathrm{X}_{3} \mathrm{X}_{5}$ $+0.40 \mathrm{X}_{4} \mathrm{X}_{5}$

Subject to:

! It starts with \#1.00

$$
X_{1}+X_{2}+X_{3}+X_{4}+X_{5}=1
$$

! We want to end with at least \#1.20

$3.71 X_{1}+2.95 X_{2}+1.61 X_{3}+1.94 X_{4}+2.56 X_{5} \geq 1.20$

! No asset may constitute more than $25 \%$ of the portfolio

$$
\begin{aligned}
& \mathrm{X}_{1}<0.25 \\
& \mathrm{X}_{2}<0.25
\end{aligned}
$$

$$
\begin{aligned}
& X_{3}<0.25 \\
& X_{4}<0.25 \\
& X_{5}<0.25
\end{aligned}
$$

The research employs LINGO software to create the Lagrangian expression. The input procedure for LINGO requires the model be converted to through linear form by writing the first order conditions. To do this we introduce Lagrangian multiplier for each constraint. There are seven (7) constraints, we shall use seven (7) dual variables devoted respectively as UNITY, RETURN, $\mathrm{X}_{1}$ FRAC, $\mathrm{X}_{2}$ FRAC, $\mathrm{X}_{3}$ FRAC, $\mathrm{X}_{4}$ FRAC, $\mathrm{X}_{5}$ FRAC.

The Lagrangian expression corresponding to this model is now

$\operatorname{MIN~R}\left(\mathrm{X}_{1}, \mathrm{X}_{2}, \mathrm{X}_{3}, \mathrm{X}_{4}, \mathrm{X}_{5}\right)=0.25 \mathrm{X}_{1}^{2}+0.23 \mathrm{X}_{2}^{2}+0.45 \mathrm{X}_{3}^{2}$ $+0.87 \mathrm{X}_{4}^{2}+0.43 \mathrm{X}_{5}^{2}+0.18 \mathrm{X}_{1} \mathrm{X}_{2}+0.12 \mathrm{X}_{1} \mathrm{X}_{3}-0.20 \mathrm{X}_{1} \mathrm{X}_{4}+$ $0.03 X_{1} X_{5}+0.07 X_{2} X_{3}-0.27 X_{2} X_{4}-0.06 X_{2} X_{5}-0.11 X_{3} X_{4}-$ $0.13 \mathrm{X}_{3} \mathrm{X}_{5}+0.40 \mathrm{X}_{4} \mathrm{X}_{5}+\left(\mathrm{X}_{1}+\mathrm{X}_{2}+\mathrm{X}_{3}+\mathrm{X}_{4}+\mathrm{X}_{5}\right) \mathrm{UNITY}$ $+\left[1.20-\left(3.71 \mathrm{X}_{1}+2.95 \mathrm{X}_{2}+1.61 \mathrm{X}_{3}+1.94 \mathrm{X}_{4}+\right.\right.$ 2.56X5)RETURN + ( $\left.X_{1}-0.25\right) X_{1}$ FRAC $+\left(X_{2}-0.25\right)$ $\mathrm{X}_{2} \mathrm{FRAC}+\left(\mathrm{X}_{3}-0.25\right) \mathrm{X}_{3}$ FRAC $+\left(\mathrm{X}_{5}-0.25\right) \mathrm{X}_{5}$ FRAC

Next to compute the first order conditions $\frac{\partial R}{\partial X 1}=0.5 \mathrm{X}_{1}+0.18 \mathrm{X}_{2}+0.12 \mathrm{X}_{3}-0.20 \mathrm{X}_{4}+0.03 \mathrm{X}_{5}+$
$\mathrm{UNITY}-3.71 \mathrm{RETURN}+\mathrm{X}_{1}$ FRAC $>0$

$\frac{\partial R}{\partial X 2}=0.18 \mathrm{X}_{1}+0.46 \mathrm{X}_{2}+0.07 \mathrm{X}_{3}-0.27 \mathrm{X}_{4}-0.06 \mathrm{X}_{5}+$ UNITY -7.95 RETURN $+X_{5}$ FRAC $>0$

$\frac{\partial R}{\partial X a}=0.12 \mathrm{X}_{1}+0.07 \mathrm{X}_{2}+0.9 \mathrm{X}_{3}-0.11 \mathrm{X}_{4}-0.13 \mathrm{X}_{5}+$ UNITY -1.61 RETURN $+\mathrm{X}_{3}$ FRAC $>0$

$\frac{\partial R}{\partial X 4}=-0.20 \mathrm{X}_{1}-0.27 \mathrm{X}_{2}-0.11 \mathrm{X}_{3}+1.74 \mathrm{X}_{4}+0.40 \mathrm{X}_{5}$ + UNITY -1.94 RETURN $+\mathrm{X}_{4}$ FRAC $>0$

$\frac{\partial R}{\partial X 5}=0.03 \mathrm{X}_{1}-0.06 \mathrm{X}_{2}-0.13 \mathrm{X}_{3}+0.40 \mathrm{X}_{4}+0.86 \mathrm{X}_{5}+$ UNITY -2.56 RETURN $+\mathrm{x}_{5}$ FRAC $>0$

$\frac{\partial R}{\partial \text { UNITY }}=\mathrm{X}_{1}+\mathrm{X}_{2}+\mathrm{X}_{3}+\mathrm{X}_{4}+\mathrm{X}_{5}-1$

$\frac{\partial R}{\partial \operatorname{RETURN}}=1.20\left(\mathrm{E}_{\mathrm{x} 1} \mathrm{X}_{1}+\mathrm{E}_{\mathrm{x} 2} \mathrm{X}_{2}+\mathrm{E}_{\mathrm{x} 3} \mathrm{X}_{3}+\mathrm{E}_{\mathrm{x} 4} \mathrm{X}_{4}+\mathrm{E}_{\mathrm{x} 5} \mathrm{X}_{5}\right)$

Adding the real constraints 


\section{International Journal of Engineering Technology and Management Sciences}

Website: ijetms.in Issue: 7 Volume No.4 November -2020 DOI: 10.46647/ijetms.2020.v04i07.001

$$
\begin{aligned}
& X_{1}+X_{2}+X_{3}+X_{4}+X_{5}=1 \\
& 3.71 X_{1}+2.95 X_{2}+1.61 X_{3}+1.94 X_{4}+2.56 X_{5} \geq 1.20 \\
& X_{1}<0.25 \\
& X_{2}<0.25 \\
& X_{3}<0.25 \\
& X_{4}<0.25 \\
& X_{5}<0.25
\end{aligned}
$$

The final model is

Min $\mathrm{X}_{1}+\mathrm{X}_{2}+\mathrm{X}_{3}+\mathrm{X}_{4}+\mathrm{X}_{5}+\mathrm{UNITY}+\mathrm{RETURN}+$ $\mathrm{X}_{1}$ FRAC $+\mathrm{X}_{2}$ FRAC $+\mathrm{X}_{3}$ FRAC $+\mathrm{X}_{4}$ FRAC $+\mathrm{X}_{5}$ FRAC

I First order condition for $\mathrm{X}_{1}$ :

$0.5 \mathrm{x}_{1}+0.18 \mathrm{x}_{2}+0.12 \mathrm{x}_{3}-0.20 \mathrm{x}_{4}+0.03 \mathrm{x}_{5}+\mathrm{UNITY}-3.71$ RETURN $+\mathrm{X}_{1}$ FRAC $>0$

I First order condition for $\mathrm{X}_{2}$ :

$0.18 \mathrm{X}_{1}+0.46 \mathrm{X}_{2}+0.07 \mathrm{X}_{3}-0.27 \mathrm{X}_{4}-0.06 \mathrm{X}_{5}+\mathrm{UNITY}-$ 2.95 RETURN $+\mathrm{X}_{2}$ FRAC $>0$

! First order condition for $\mathrm{X}_{3}$ :

$0.12 \mathrm{X}_{1}+0.07 \mathrm{X}_{2}+0.93 \mathrm{X}_{3}-0.11 \mathrm{X}_{4}-0.13 \mathrm{X}_{5}+\mathrm{UNITY}-$ 1.61 RETURN $+X_{3}$ FRAC $>0$

I First order condition for $\mathrm{X}_{4}$ :

$-0.20 \mathrm{X}_{1}-0.27 \mathrm{X} 2-0.11 \mathrm{X} 3+1.74 \mathrm{X}_{4}+040 \mathrm{X}_{5}+\mathrm{UNITY}-$ 1.94 RETURN $+\mathrm{X}_{4}$ FRAC $>0$

! First order condition for $\mathrm{X}_{5}$ :

$0.03 \mathrm{X}_{1}-0.06 \mathrm{X}_{2}-0.13 \mathrm{X}_{3}+0.40 \mathrm{X}_{4}+0.86 \mathrm{X}_{5}+\mathrm{UNITY}-$ 2.56 RETURN $+\mathrm{X}_{5}$ FRAC $>0$

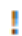
Start of "real" constraints

I Budget Constraint, multiplier is UNITY.

$$
\mathrm{X}_{1}+\mathrm{X}_{2}+\mathrm{X}_{3}+\mathrm{X}_{4}+\mathrm{X}_{5}=1
$$

IGrowth constraint, multiplier is RETURN:

$3.7 X_{1}+2.95 X_{2}+1.61 X_{3}+1.94 X_{4}+2.56 X_{5}>1.20$

I Max Fraction of $\mathrm{X}_{1}$, multipliers is $\mathrm{X}_{1}$ FRAC:

$$
\mathrm{X}_{1}<.25
$$

I Max Fraction of $\mathrm{X}_{2}$, multipliers is $\mathrm{X}_{2}$ FRAC:

$$
\mathrm{X}_{2}<.25
$$

! Max Fraction of $\mathrm{X}_{3}$, multipliers is $\mathrm{X}_{3}$ FRAC:

$$
\mathrm{X}_{3}<.25
$$

I Max Fraction of $\mathrm{X}_{4}$, multipliers is $\mathrm{X}_{4}$ FRAC:

$$
\mathrm{X}_{4}<.25
$$

I Max Fraction of $\mathrm{X}_{5}$, multipliers is $\mathrm{X}_{5}$ FRAC:

$$
\begin{aligned}
& \mathrm{X}_{5}<.25 \\
& \text { END } \\
& \text { QCP } 7
\end{aligned}
$$

VI. RESULT OF THE MODEL OBTAINED FROM LINDO SOFTWARE

\section{AT $20 \%$} LP OPTIMUM FOUND AT STEP 5 OBJECTIVE FUNCTION VALUE

1) $\quad 1.000000$

\section{VARIABLE VALUE REDUCED COST}

$\begin{array}{lcc}\text { X1 } & 0.200000 & 0.000000 \\ \text { X2 } & 0.200000 & 0.000000 \\ \text { X3 } & 0.200000 & 0.000000 \\ \text { X4 } & 0.200000 & 0.000000 \\ \text { X5 } & 0.200000 & 0.000000 \\ \text { UNITY } & 0.000000 & 1.000000 \\ \text { RETURN } & 0.000000 & 1.000000 \\ \text { X1FRAC } & 0.000000 & 1.000000 \\ \text { X2FRAC } & 0.000000 & 1.000000 \\ \text { X3FRAC } & 0.000000 & 1.000000 \\ \text { X4FRAC } & 0.000000 & 1.000000 \\ \text { X5FRAC } & 0.000000 & 1.000000\end{array}$

AT $21 \%$

LP OPTIMUM FOUND AT STEP 5

OBJECTIVE FUNCTION VALUE 


\section{International Journal of Engineering Technology and Management Sciences}

Website: ijetms.in Issue: 7 Volume No.4 November - 2020 DOI: 10.46647/ijetms.2020.v04i07.001

$\begin{array}{ccc}1.000000 & & \\ \text { VARIABLE } & \text { VALUE } & \text { REDUCED COST } \\ \text { X1 } & 0.210000 & 0.000000 \\ \text { X2 } & 0.210000 & 0.000000 \\ \text { X3 } & 0.210000 & 0.000000 \\ \text { X4 } & 0.160000 & 0.000000 \\ \text { X5 } & 0.210000 & 0.000000 \\ \text { UNITY } & 0.000000 & 1.000000 \\ \text { RETURN } & 0.000000 & 1.000000 \\ \text { X1FRAC } & 0.000000 & 1.000000 \\ \text { X2FRAC } & 0.000000 & 1.000000 \\ \text { X3FRAC } & 0.000000 & 1.000000 \\ \text { X4FRAC } & 0.000000 & 1.000000 \\ \text { X5FRAC } & 0.000000 & 1.000000\end{array}$

NO. ITERATIONS $=5$

AT $22 \%$
LP OPTIMUM FOUND AT STEP 5
OBJECTIVE FUNCTION VALUE
$\begin{array}{ccc}\text { 1) } 1.000000 & \\ \text { VARIABLE } & \text { VALUE } & \text { REDUCED COST } \\ \text { X1 } & 0.220000 & 0.000000 \\ \text { X2 } & 0.220000 & 0.000000 \\ \text { X3 } & 0.220000 & 0.000000 \\ \text { X4 } & 0.120000 & 0.000000 \\ \text { X5 } & 0.220000 & 0.000000 \\ \text { UNITY } & 0.000000 & 1.000000 \\ \text { RETURN } & 0.000000 & 1.000000 \\ \text { X1FRAC } & 0.000000 & 1.000000\end{array}$

$\begin{array}{lrr}\text { X2FRAC } & 0.000000 & 1.000000 \\ \text { X3FRAC } & 0.000000 & 1.000000 \\ \text { X4FRAC } & 0.000000 & 1.000000 \\ \text { X5FRAC } & 0.000000 & 1.000000 \\ \text { NO. ITERATIONS }=5 & \\ \text { AT } 23 \%\end{array}$

LP OPTIMUM FOUND AT STEP 5

OBJECTIVE FUNCTION VALUE

1) $\quad 1.000000$

VARIABLE VALUE REDUCED COST

$\begin{array}{lll}\mathrm{X} 1 & 0.230000 & 0.000000\end{array}$

$\begin{array}{lll}\mathrm{X} 2 & 0.230000 & 0.000000\end{array}$

$\begin{array}{lll}\mathrm{X} 3 & 0.230000 \quad 0.000000\end{array}$

$\begin{array}{lll}\mathrm{X} 4 & 0.080000 \quad 0.000000\end{array}$

$\begin{array}{lll}\mathrm{X} 5 & 0.230000 & 0.000000\end{array}$

UNITY $\quad 0.000000 \quad 1.000000$

RETURN $\quad 0.000000 \quad 1.000000$

X1FRAC $\quad 0.000000 \quad 1.000000$

X2FRAC $\quad 0.000000 \quad 1.000000$

$\begin{array}{lll}\text { X3FRAC } & 0.000000 & 1.000000\end{array}$

$\begin{array}{lll}\text { X4FRAC } & 0.000000 & 1.000000\end{array}$

$\begin{array}{lll}\text { X5FRAC } & 0.000000 & 1.000000\end{array}$

NO. ITERATIONS $=5$

AT $24 \%$

LP OPTIMUM FOUND AT STEP 5

OBJECTIVE FUNCTION VALUE

1) 1.000000

$\begin{array}{crr}\text { VARIABLE } & \text { VALUE } & \text { REDUCED COST } \\ \mathrm{X} 1 & 0.240000 & 0.000000\end{array}$ 


\section{International Journal of Engineering Technology and Management Sciences}

Website: ijetms.in Issue: 7 Volume No.4 November - 2020 DOI: 10.46647/ijetms.2020.v04i07.001

$\begin{array}{ccc}\text { X2 } & 0.240000 & 0.000000 \\ \text { X3 } & 0.240000 & 0.000000 \\ \text { X4 } & 0.040000 & 0.000000 \\ \text { X5 } & 0.240000 & 0.000000 \\ \text { UNITY } & 0.000000 & 1.000000 \\ \text { RETURN } & 0.000000 & 1.000000 \\ \text { X1FRAC } & 0.000000 & 1.000000 \\ \text { X2FRAC } & 0.000000 & 1.000000 \\ \text { X3FRAC } & 0.000000 & 1.000000 \\ \text { X4FRAC } & 0.000000 & 1.000000 \\ \text { X5FRAC } & 0.000000 & 1.000000\end{array}$

available fund by investors should be allocated to available investment open to investors. It determined that all return on asset of the insurance companies (Linkage, Niger, Mutual Benefit, LASACO and AIICO) contribute to the investor's return.

Finally, for a good product mixed or investment, equal percentage of investor's capital should be invest on Linkage insurance company, Mutual Benefit insurance company, Niger insurance company, LASACO insurance company and other remaining percent should be allocated to AIICO insurance company, so as to maximize the investor's return.

\section{NO. ITERATIONS $=5$}

Table2: The summary of the results yield the table below for the purpose of comparison and decisions

\begin{tabular}{|l|l|l|l|l|l|l|l|}
\hline $\mathrm{T}$ & $\mathrm{X} 1$ & $\mathrm{X} 2$ & $\mathrm{X} 3$ & $\mathrm{X} 4$ & $\mathrm{X} 5$ & Variance & $\begin{array}{l}\text { LP } \\
\text { optimum } \\
\text { step }\end{array}$ \\
\hline 1.20 & 0.200000 & 0.200000 & 0.200000 & 0.200000 & 0.200000 & 1.000000 & 5 \\
\hline 1.21 & 0.210000 & 0.210000 & 0.210000 & 0.160000 & 0.210000 & 1.000000 & 5 \\
\hline 1.22 & 0.220000 & 0.220000 & 0.220000 & 0.120000 & 0.220000 & 1.000000 & 5 \\
\hline 1.23 & 0.230000 & 0.230000 & 0.230000 & 0.80000 & 0.230000 & 1.000000 & 5 \\
\hline 1.24 & 0.240000 & 0.240000 & 0.240000 & 0.40000 & 0.240000 & 1.000000 & 5 \\
\hline
\end{tabular}

\section{DISCUSSION OF RESULTS}

The increment that yield the minimum percent with mixed investment opportunity is $4 \%$. Hence the optimum solution to the model is $\mathrm{X}_{1}=24 \%, \mathrm{X}_{2}=24 \%, \mathrm{X}_{3}=24 \%, \mathrm{X}_{4}=4 \%$, and $X_{5}=24 \%$

\section{CONCLUSION}

This research shows how portfolio selection of return on assets of the five selected insurance company in Nigeria was used the past financial records of each insurance company for ten years. Also, it shows how allocation of

\section{REFERENCES}

[1] Ali yousfat (2015).The Portfolio Selection by Using Quadratic Programming Approach Case Study of Malaysia Stock Exchange. International Journal of Engineering and Technology (IJET), Vol 7 No 4,Pp. 1361-1369.

[2] David G. Luenberger (2008). Linear and non-linear Programming, Springer Verlag New York.

[3] Ebraham Zam (2008) Modern Portfolio Theory 2008 (http en Wikipedia. Org) 
International Journal of Engineering Technology and Management Sciences

Website: ijetms.in Issue: 7 Volume No.4 November - 2020 DOI: 10.46647/ijetms.2020.v04i07.001

[4] Emiola, O.K.S, and Adeoye A.O (2014) Analysis of Bonus on share as a Determinant for Portfolio Selection of Bank Share. International Journal of Advanced Research in Computer Science. Volume 5, No. 8, Nov Dec; 2014 ISSN No 0976 - 5697 Pg 1 - 4. Available Online at www.iircs.info

[5] Emiola, O.K.S, and Adeoye A.O (2014) Return on Invested Capital as a Determinant for Future Investment. International Journal of Advanced Research in Computer Science. Volume 5, No. 8, Nov - Dec; 2014 ISSN No 0976 - 5697 Pg 54 - 57. Available Online at ww wire s .info

[6] Emiola O.K.S. and Alayemi S.A (2015) Dividend on Share as a Policy for Portfolio Slection of Bank Share, /pas/ international Journal of Computer Science (11JCS). Volume 3, Issue 5. May 2015. Pg 18 - 24 ISSN $2321 \quad-\quad 5992 . \quad$ Available Online at http/Avww.ipasj.org/ 11JCS/ 11JCS. Htm

[7] Emiola O.K.S. and Alayemi S.A (2015) Using Dividend Payout Ratio as a Determinant for Portfolio Selection of Bank Share. International Journal of Management (11JM), Volume 3, Issue 2, February 2015 Pg 20 - 25, ISSN $2321-645 \mathrm{X}$. Available Online at http//www.ipasj.org/11JM/1 lJM.htm.

[8] Etukudo I. A, Effanga E. O, Onwukwe C. E and Umoren M. U (2009) Application of portfolio selection model for optimal allocation of investible funds in a portfolio

[9] Etukudo I. A and Umoren M. U (2009). A comparison of a modified super convergent line series algorithm and modified simplex method for solving quadratic programming ICASTOR journal of mathematical sciences Kolkata India Vol 3 No 1 41-61

[10] Hillier F. C and liebemram G. J (2006) Introduction to operation research Eight Edition. Tata McGraw - Hill New DEllin

[11] Kothari C. R (2004) Research methodology methods and techniques $2^{\text {nd }}$ Edition NEW AGE INTERNATIONAL (P) LIMITED INDIA

[12] Markowitz, H.M (1959) Portfolio Selection: Efficient Diversification of Investments. New York: John Wiley $\&$ Sons.

[13] Prem K. G. and Hira .D. S. (2009): Operation Research S. Chand \& Company Ltd. New Delhi.

[14] Sharma J. K (2013) Operation Research theory and application. $5^{\text {th }}$ Edition MACMILLAN PUBLISHERS INDIA LIMITED. 